\section{COMMUNITY BASED STUDY ON FAMILY RELATED CONTRIBUTORY FACTORS FOR CHILDHOOD UNINTENTIONAL INJURIES IN AN URBAN SETTING OF SRI LANKA}

DH Punyadasa, D Samarakkody. Ministry of Health, Sri Lanka

\subsection{6/injuryprev-2016-042156.696}

Background Childhood unintentional injuries are unexpected occurrences affecting a child, which lead to immeasurable grief and suffering to entire family. It is one of the leading causes of hospitalisation among children in Sri Lanka resulting death and disability. Children aged 1-4 years are at greater risk of unintentional injuries and majority of injuries among this age group occur in their family environment. Prevention of such injuries requires a clear understanding of their contributory factors.

Methods A community based descriptive cross sectional study was carried out among children aged 1-4 years residing in an urban setting of Sri Lanka, to assess the incidence and associated family related factors of unintentional injuries. 458 children were recruited using simple random sampling technique, giving a response rate of $91.6 \%$. Parents of selected children completed an interviewer administered questionnaire (IAQ) that recorded the parent's recollection of their child having an unintentional injury needed medical attention, within three months prior to the interview. Socio demographic factors and family related factors of the study subjects were also collected by the IAQ. The data was analysed using SPSS 18.2 statistical package.

Results The incidence of unintentional injuries during the study period of three months was 28.1 per 100 children $(95 \%$ CI = 19.46-36.74).

The factors that were significantly associated with the occurrence of unintentional injuries among children are low monthly income of the family ( $\mathrm{p}=0.045$ ), low social support to the mother of index child $(\mathrm{p}=0.022)$, non authoritative type of parenting of the mother of index child $(p=0.039)$, cared by person other than mother during day time $(\mathrm{p}=0.002)$, frequent arguments between parents $(\mathrm{p}=0.004)$ and frequent alcohol consumption of father $(\mathrm{p}=0.001)$.

Conclusions Unintentional injuries are an important child health issue among children aged 1-4 years. Identification of family related contributory factors for unintentional injuries among this age group will enable policy makers to formulate effective child injury preventive interventions.

\section{THE IMPORTANCE OF BIKE'S BRAKE ADJUSTMENT}

${ }^{1}$ Kimiko Deguchi, 2,3 Koji Kitamura, 2,3 Yoshifumi Nishida, ${ }^{2,3}$ Mikiko Oono, ${ }^{4,2,3}$ Tatsuhiro Yamanaka. ${ }^{1}$ Deguchi PaediatricClinic, Japan; ${ }^{2}$ National Institute of Advanced Industrial Science and Technology, Japan; ${ }^{3}$ Safe Kids Japan, Japan ; Ryokuen Children's Clinic, Japan

10.1136/injuryprev-2016-042156.697

Background Bicycle crashes are a major cause of injuries in childhood. According to injury data from the Bodygraphic Injury Surveillance System of the National Centre for Child Health and Development, bicycles are the number one product related to childhood injuries. Although it is important to keep one's brakes adjusted to prevent bicycle-related injuries, less attention has been paid to its importance compared to a helmet use. The purpose of this study is to measure the characteristics of children's hand growth and to reveal the relationship between a child's hand size and a reaction time to brake.
Methods Ten boys and 10 girls from each grade (120 students in total) participated in this study. To gather school children's anthropometric hand data, we measured the palm length, the middle finger length, and the length between the base of the thumb and the DIP of a middle finger. For the reaction time measurement, we prepared two bikes which attached the different size of width (standard or wide). Each participant rode both bikes and measured their reaction time. More specifically, the reaction time means the time from a participant notices the sign to stop to the time when the brake in the rear wheel begins to work. We considered the standard width as fit of one's hand size. Results The length of both the palm and the middle finger increased 1.2 times in 6 years of elementary school. The growth rates were bigger among students in 4, 5, 6 grades compared to students in 1, 2, 3 grades. When the brake lever width does not fit one's hand, the reaction time to brake is delayed by $0.1 \mathrm{sec}-$ ond. Moreover, we found the tendency that one required more reaction time to brake if one's hand size is small.

Conclusions We revealed the relationship between a child's hand size and a reaction time to brake and the importance of bike's brake adjustment. Based on the results from this study, we developed an animated video for bike safety. In future studies, we planned to implement an educational program to recommend a regular bike maintenance including a bike brake in addition to a helmet use.

\section{DEALING WITH DIVERSITY: CHILD INJURY PREVENTION STRATEGIES FOR A CULTURALLY DIVERSE WESTERN AUSTRALIA}

Katherine Celenza. Kidsafe Western Australia, Australia

\subsection{6/injuryprev-2016-042156.698}

Background Western Australia cannot be a one size fits all when it comes to child injury prevention, the size of the state and diversity of each region makes it important to have targeted and appropriate campaigns and resources. Perth is one of the most remote cities and the extent of the Western Australian state means that accessing parents and carers that are remote and isolated takes an important role in ensuring that resources and programs are targeted and specific to each region.

Description of problem Addressing a shortage of culturally appropriate information available for parents and carers of children on the prevention of common childhood injuries was the purpose of this program. Ensuring that messaging and resources specifically targeted high risk regional Aboriginal communities, were culturally appropriate and regionally specific to address the issue of child injury prevention in Western Australia. Kidsafe WA worked with individual regional and remote Aboriginal communities to adapt current programs and resources to specifically address each region and the injury issues associated. Ensuring messaging was specific, culturally appropriate and addressed local issues was key to targeting a diverse area.

Results This paper will outline what was learned through the consultation process undertaken with community members within regional Aboriginal communities throughout the Pilbara, Kimberley and Goldfields Region. It will focus on the strengths and weaknesses of existing practices, how the program has been evaluated and what the next step is in addressing the issues raised for reducing child injuries.

Conclusions The progression of this project towards a suite of resources available across WA has reinforced the need for 